\title{
Evaluation of C-reactive protein, Haptoglobin and cardiac troponin 1 levels in brachycephalic dogs with upper airway obstructive syndrome
}

\author{
Marta Planellas ${ }^{1 *}$, Rafaela Cuenca ${ }^{1}$, Maria-Dolores Tabar ${ }^{2}$, Coralie Bertolani ${ }^{3}$, Cyrill Poncet ${ }^{3}$, Josep M Closa ${ }^{4}$, \\ Juan Lorente ${ }^{5}$, Jose J Cerón ${ }^{6}$ and Josep Pastor ${ }^{1}$
}

\begin{abstract}
Background: Brachycephalic dogs have unique upper respiratory anatomy with abnormal breathing patterns similar to those in humans with obstructive sleep apnea syndrome (OSAS). The objective of this study was to evaluate the correlation between anatomical components, clinical signs and several biomarkers, used to determine systemic inflammation and myocardial damage (C-reactive protein, CRP; Haptoglobin, Hp; cardiac troponin I, CTnl), in dogs with brachycephalic upper airway obstructive syndrome (BAOS).

Results: Fifty brachycephalic dogs were included in the study and the following information was studied: signalment, clinical signs, thoracic radiographs, blood work, ECG, components of BAOS, and CRP, Hp and cTnl levels. A high proportion of dogs with BAOS (88\%) had gastrointestinal signs. The prevalence of anatomic components of BAOS was: elongated soft palate (100\%), stenotic nares (96\%), everted laryngeal saccules (32\%) and tracheal hypoplasia (29.1\%). Increased serum levels of biomarkers were found in a variable proportion of dogs: $14 \%$ (7/50) had values of CRP $>20 \mathrm{mg} / \mathrm{L}$, 22.9\% (11/48) had values of $\mathrm{Hp}>3 \mathrm{~g} / \mathrm{L}$ and 47.8\% (22/46) had levels of CTnl > $0.05 \mathrm{ng} /$ $d$ l. Dogs with everted laryngeal saccules had more severe respiratory signs $(p<0.02)$ and higher values of CRP $(p<0.044)$. No other statistical association between biomarkers levels and severity of clinical signs was found.

Conclusions: According to the low percentage of patients with elevated levels of CRP and Hp, BAOS does not seem to cause an evident systemic inflammatory status. Some degree of myocardial damage may occur in dogs with BAOS that can be detected by cTnl concentration.
\end{abstract}

Keywords: Acute phase proteins, Apnea, Brachycephalic, Hypoxia, Myocardial damage, Upper airway

\section{Background}

Brachycephalic upper airway obstructive syndrome (BAOS) is a combination of nasal and oropharyngeal anatomic abnormalities resulting from selective breeding to reduce the length of the maxilla without concurrent reduction in the soft tissue of the nose, palate, and pharynx $[1,2]$. The resulting excess of soft tissue causes airway obstruction in affected animals, with clinical signs that may include inspiratory stertor and stridor, exercise and heat intolerance, cyanosis, respiratory distress, regurgitation, and vomiting. The primary abnormalities of

\footnotetext{
*Correspondence: marta.planellas@uab.cat

${ }^{1}$ Animal Medicine and Surgery Department. Faculty of Veterinary Medicine, Universitat Autònoma de Barcelona (UAB), Cerdanyola del Vallès, Barcelona 08193, Spain

Full list of author information is available at the end of the article
}

BAOS are stenotic nares and an overlong soft palate. Secondary changes that occur as a result of chronic upper airway obstruction include eversion of laryngeal saccules, pharyngeal edema and laryngeal collapse. Brachycephalic dogs can present other respiratory tract abnormalities such as tracheal hypoplasia, bronchial collapse, macroglossia, reduced buccal opening, nasopharyngeal collapse and nasopharyngeal turbinates [1-7]. Such dogs have anatomic abnormalities that include small pharyngeal airways and abnormal breathing patterns similar to those in humans with obstructive sleep apnea syndrome (OSAS) [8,9]. On the other hand, it have been reported that the English bulldog has snoring, fragmented sleep with mild hypoxemia and apneas in the same manner as human patients with OSAS. Those 
anatomic and clinical traits, made the English bulldog a suitable natural animal model for human OSAS $[8,9]$.

Inflammation is the characteristic trait of the pathophysiology of OSAS and represents a pathway linking human OSAS patients with increased cardiovascular morbidity $[10,11]$. C-reactive protein (CRP) levels have been shown to be high in the presence of hypoxia, for example at high altitude [12]. Furthermore, CRP may also increase with sleep deprivation [13]. Both hypoxemia and sleep deprivation are frequently present in human patients with OSAS, and therefore may participate in the genesis of an inflammatory response [13]. Hypoxia also may increase fatigability of upper airway dilator muscle and soft tissue, in a manner that has the potential to worsen disease. Inflammation, as well as oxidative stress, resulted from the repeated hypoxia and reoxygenations could be implicated in the injury to these tissues [14,15].

Some epidemiological studies have linked high haptoglobin (Hp) levels with a greater incidence of myocardial infarct [16]. Kim and others [16] have reported that human patients with OSAS have higher levels of $\mathrm{Hp}$, which fell after medical treatment with a continuous positive airway pressure device.

Cardiac troponin I (cTnI) and $\mathrm{T}(\mathrm{c} T \mathrm{~T} \mathrm{~T})$ are definitive biomarkers for detection of myocardial injury in human patients $[17,18]$, and more recently in dogs $[19,20]$. In dogs with acute myocardial injuries or infarction, the concentration of cTnI has been shown to be positively correlated with the size of the infarct [21]. Moreover, chronic increases in cTnI are associated with chronic or ongoing pathologic myocardial process [21].

Even though human OSAS is not equal to canine BAOS, similarities exist. On the other hand, dogs with BAOS present clinical abnormalities (chronic hypoxia, gastrointestinal signs and subclinical heatstroke) that make it worthy to rule out the presence of systemic inflammation and myocardial damages.

The objective of this study was to evaluate the correlation between anatomical components, clinical signs and several biomarkers, used to determine systemic inflammation and myocardial damage (CRP, Hp, cTnI), in dogs with BAOS.

\section{Results}

\section{Epidemiological data}

Male dogs were over-represented in the study since 30 males (two neutered) and 20 females (four neutered) were evaluated. The median age was 2.5 years old (range 1-10 years old). Thirty-eight dogs (76\%) were less than three years old. All were purebred: French bulldogs were the commonest breed studied and accounted for 29 of the 50 cases (58\%), while other breeds evaluated included English bulldogs $(\mathrm{n}=8,16 \%)$, Pug $(\mathrm{n}=12,24 \%)$ and Shih-tzu ( $\mathrm{n}=1,2 \%)$.

\section{Clinical signs}

All dogs had some kind of respiratory signs (Table 1): only one dog had a mild grade of respiratory signs (grade 1), 13 (26\%) suffered from a moderate grade (grade 2) and 36 $(72 \%)$ from a severe grade (grade 3 ). Although a large number of animals with respiratory signs also had digestive signs (mild or moderate), no correlation was found between both types of signs $(p=0.71)$.

Electrocardiograms (ECG) were recorded previously to upper airway (UA) inspection and the presence of arrhythmia and heart rate was recorded. Three out of fifty presented sinusal arrhythmia indicating parasympathetic dominance. Heart rate values ranged from 70 to 180 beats per minute. No other abnormalities have been observed in ECG reports.

The inspection of the UA respiratory tracts revealed abnormalities in all 50 patients: abnormally long and hyperplasic soft palate (50/50 dogs; $100 \%)$, stenotic nares (48/50 dogs; 96\%) and everted laryngeal saccules (16/50 dogs; 32\%). Thoracic radiography was performed in 48 cases and tracheal hypoplasia was diagnosed in 14 animals 29.1\% dogs; pug $(n=4)$, English bulldog $(n=2)$ and French bulldog $(n=8)$ ] with a DTI/DT (thoracic inlet to tracheal diameter) ratio that ranged from 0.08 to 0.3 . Thoracic radiography also allowed us to record vertebral

Table 1 Gradation of respiratory and digestive signs in $\mathbf{5 0}$ brachycephalic dogs suffering from BAOS

\begin{tabular}{|c|c|c|c|c|c|}
\hline \multirow[t]{3}{*}{ Grade of clinical signs } & \multirow{2}{*}{\multicolumn{2}{|c|}{$\begin{array}{l}\text { Respiratory signs } \\
\text { (stertors, snoring, } \\
\text { inspiratory efforts, } \\
\text { stress, exercise } \\
\text { intolerance, } \\
\text { syncope) }\end{array}$}} & \multirow[t]{3}{*}{ Grade of clinical signs } & \multirow{2}{*}{\multicolumn{2}{|c|}{$\frac{\text { Digestive signs }}{\text { (regurgitation, vomiting) }}$}} \\
\hline & & & & & \\
\hline & n & $\%$ & & $\mathrm{n}$ & $\%$ \\
\hline Grade 0- Absent & 0 & $0 \%$ & Grade 0 - Absent & 6 & $12 \%$ \\
\hline Grade 1-Mild Stertors during exercise (<once week) & 1 & $2 \%$ & Grade 1-Mild (< once week) & 23 & $46 \%$ \\
\hline Grade 2-Moderate Continuous stertor (< once daily) & 13 & $26 \%$ & Grade 2-Moderate (< once daily) & 14 & $28 \%$ \\
\hline Grade 3-Severe Exercice intolerance, cianosis (>once daily) & 36 & $72 \%$ & Grade 3-Severe (> once daily) & 7 & $14 \%$ \\
\hline
\end{tabular}


heart score (VHS) of 35 out of 50 patients, obtaining values from 8.5 to 12.1 [22]. The elevated incidence of hemivertebra in brachycephalic dogs made the VHS measurement impossible in 15 dogs.

\section{Laboratory data}

Table 2 shows the relationship between CRP, Hp and cTnI levels in the different breeds. The median CRP concentration in brachycephalic dogs with BAOS was $7.8 \mathrm{mg} / \mathrm{L}$ (3.6-235 mg/L). Seven of the 50 dogs studied had high concentrations of CRP. Hp levels were measured in 48 dogs, with a median $\mathrm{Hp}$ concentration of $1.6 \mathrm{~g} / \mathrm{L}$ (range 0.09-13.9 g/L). From those, 11 (22.9\%) had increased Hp levels. The median cTnI value was $0.05 \mathrm{ng} / \mathrm{ml}(<0.05-0.29)$, while $47.8 \%(22 / 46)$ of dogs with BAOS had high values of cTnI $(>0.05)$.

Table 3 shows that there was a significant correlation between the severity of the respiratory signs and male patients $(p=0.026)$.

The present study did not find a significant association between the severity of respiratory and digestive signs and CRP, Hp and cTnI levels. However, patients with everted laryngeal saccules had more severe respiratory signs $(p=0.02)$ and higher levels of CRP $(p=0.044)$. The presence of tracheal hypoplasia was not associated with an increased severity of respiratory signs.

There was a significant correlation between the presence of digestive signs and $c \operatorname{TnI}(p=0.016)$. When the severity digestive signs was studied in relation to cTnI levels, it was observed that animals with grade 2 of digestive signs had higher levels of cTnI than animals with grade $1(p=0.026)$. The possible relation of heart rate and arrhythmias with CRP, Hp and cTnI was statistically studied without obtaining any significance. In the same way, VHS values were not statistically associated with any biomarker (CRP, Hp and CTnI) or ECG values.

\section{Discussion}

To the authors' knowledge, this is the first prospective clinical study of dogs suffering from BAOS that has evaluated the levels of several biomarkers [acute phase proteins (CRP, Hp) and cTnI] and the relation of them with clinical signs and anatomical features.

Table 2 CRP, Hp and CTnl levels in the different breeds of the $\mathbf{5 0}$ brachycephalic dogs studied

\begin{tabular}{|c|c|c|c|c|c|c|}
\hline \multirow[t]{2}{*}{ Breed } & \multicolumn{2}{|c|}{$\mathrm{CRP}>20 \mathrm{mg} / \mathrm{dl}$} & \multicolumn{2}{|c|}{$\mathrm{Hp}>3 \mathrm{~g} / \mathrm{L}$} & \multicolumn{2}{|c|}{$\mathrm{cTnl}>0.05 \mathrm{ng} / \mathrm{dl}$} \\
\hline & $n$ & $\%$ & $n$ & $\%$ & $n$ & $\%$ \\
\hline English B & $3 / 8$ & 37.5 & $0 / 8$ & 0 & $8 / 8$ & 100 \\
\hline French B & $3 / 29$ & 10.3 & $5 / 27$ & 18.5 & $14 / 26$ & 53.8 \\
\hline Pug & $1 / 12$ & 8.3 & $6 / 12$ & 50 & $0 / 11$ & 0 \\
\hline Shitzu & $0 / 1$ & 0 & $0 / 1$ & 0 & $0 / 1$ & 0 \\
\hline Total & $7 / 50$ & 14 & $11 / 48$ & 22.9 & $22 / 46$ & 47.8 \\
\hline
\end{tabular}

Table 3 Statistical analysis showing the association of different variables with respiratory and digestive signs

\begin{tabular}{lccccc}
\hline Variable & \multicolumn{2}{c}{ Respiratory signs } & & \multicolumn{2}{c}{ Digestive signs } \\
\cline { 2 - 3 } \cline { 6 - 6 } & $\partial$ & $\boldsymbol{p}$ value & & $\partial$ & $\boldsymbol{p}$ value \\
\hline Age & 0.154 & 0.255 & & 0.052 & 0.645 \\
Sex & $-0.317^{*}$ & $0.026^{*}$ & & -0.162 & 0.258 \\
Breed & 0.197 & 0.17 & & -0.254 & 0.067 \\
HTC & -0.003 & 0.990 & & -0.131 & 0.99 \\
WBC & -0.099 & 0.583 & & -0.016 & 0.916 \\
Platelets & 0.008 & 0.97 & & -0.039 & 0.8 \\
Total Protein & 0.347 & 0.066 & & 0.206 & 0.28 \\
Ratio DT/DTI & -0.187 & 0.195 & & -0.127 & 0.38 \\
CRP & 0.076 & 0.581 & & -0.063 & 0.66 \\
Hp & -0.025 & 0.87 & & -0.143 & 0.33 \\
cTnl & 0.261 & 0.087 & $0.352^{*}$ & $0.016^{*}$ \\
\hline
\end{tabular}

The English bulldog has snoring and fragmented sleep [23] with mild hypoxemia and apneas only during rapid eye movement. English bulldog is the closest animal model for OSAS. Even if, UA obstructive disease in dogs is not equal to OSAS, important similarities exist. Some studies used brachycephalic dogs, as an animal model, to evaluate pharyngeal dilator muscular damages in UA obstructive disease, observing similar alterations to human patients with OSAS [23,24].

The correlation between human OSAS, inflammation and cardiovascular disease is well established $[10,13,16]$. The mechanism involved in the development of cardiovascular disease is attributed to increased sympathetic activity due to sleep deprivation and fragmentation, and repetitive hypoxemic events with significant arterial desaturation. These factors are believed to lead to an activation of pro-inflammatory cytokines. Even if OSAS and BAOS are different syndromes, this study tries to clear up a possible association of BAOS, inflammation and cardiovascular diseases.

The presence of digestive signs in dogs with BAOS has been described elsewhere [3,25-27]. The relationship between respiratory and digestive signs is due to the exaggerated repetitive variation of diaphragmatic pressure, present in patients with obstructive breathing, which causes gastroesophageal reflux and inflammation of the oropharyngeal areas that, in turn, worsen respiratory signs [3,27]. Poncet and others [3,27] found an association between the severity of respiratory and digestive signs in dogs with BAOS, which suggests that these signs are related. These authors also used endoscopy and histological studies to reveal the presence of lesions in the digestive tract of all dogs with BAOS. The majority of dogs with BAOS in the present study had digestive signs, but no association between the severity of digestive and respiratory signs was found. Even though the 
physiological relation of respiratory and digestive signs is clear, a correlation with severity of both signs is not always present. The results of the study indicate a correlation between the presence of digestive signs and high cTnI values, but authors did not find any plausible explanation for this association. Further studies should be performed in order to find the physiological reason of this correlation.

The most common anomalies present in dogs from the present study were stenotic nares and elongated soft palate, similar to findings described by previous studies $[16,26,28]$. However, the prevalence of everted laryngeal saccules in dogs of the present study was lower that previously reported $[4,6,29]$. Due to the prospective nature of this study, the presence of tracheal hypoplasia was evaluated in nearly all the cases included (96\%) and was found in 14 dogs $(29.1 \%)$. Tracheal hypoplasia is a concurrent finding in many patients with BAOS [1-3,5$7,25,30]$. English bulldogs are more commonly affected by tracheal hypoplasia than other breeds, which could have biased our results $[1,30,31]$. But, according other studies and our results, in the absence of concurrent pulmonary disease, tracheal hypoplasia is usually not associated with clinical signs [31-33]. It may exacerbate the respiratory signs associated with BAOS because of increased resistance to airflow, but tracheal hypoplasia, according our results and other reports, is not associated with worse outcomes after surgical correction of BAOS $[1,6,31,33,34]$.

The presence of nasopharyngeal turbinates can be an important anatomic component of BAOS [4], but in the present study it has not been evaluated in all dogs due to the multicentric nature of the study.

The innovative aspects of this work are the determination of different biomarkers in dogs with BAOS. The design of the study included a complete work-up to exclude other diseases that might affect the results of biomarker determination. In human medicine, high values of C-reactive protein (CRP) have been found in OSAS patients and have been associated with the severity of respiratory signs according to the apnea/hypopnea index [35]. The apnea/hypopnea index, obtained during polisomnography, is an important parameter for describing the severity of OSAS and describes the number of apneas or hypopneas per hour. Moreover levels of CRP, Hp and IL-6 in human patients with OSAS decreased after surgical or medical treatment $[16,36]$. A study performed with 17 brachycephalic dogs showed that some proinflammatory cytokines and nitric oxide are increased in brachycephalic dogs and also correlated with the severity of clinical signs [37]. Even though, in the present study few dogs had high values of CRP and $\mathrm{Hp}$, and no correlation with the severity of respiratory signs was observed, with the exception of patients with everted laryngeal saccules. The presence of everted laryngeal saccules is considered a secondary change from airway obstruction, but can contribute to aggravate obstructive upper airway signs. A significant association has been found between the presence of everted laryngeal, severity of respiratory signs and CRP levels indicating that the presence of everted laryngeals can increase upper airway obstruction leading to severe respiratory signs and increase of inflammatory biomarkers. By contrast, the present results suggest that CRP and Hp determination, in general, do not offer valuable information in dogs with BAOS. Probably, in brachycephalic dogs, BAOS do not cause such an inflammatory status as OSAS in human patients. In human medicine, OSAS is diagnosed in adult patients and anatomical abnormalities are not the unique etiology of the syndrome. On the other hand, BAOS is diagnosed in young brachycephalic patients with upper airway abnormalities. Maybe those differences justify a less obvious systemic inflammatory response in dogs suffering from BAOS. Actually, a clinical grade system and upper airway examination can define BAOS in canine patients. Even though, an objective clinical test, such as apnea-hipopnea index, obtained during polisomnography and used to define human OSAS severity, could offer valuable information to better classify and understand BAOS. On the other hand, the respiratory functional assessment using barometric whole-body plethysmography in brachycephalic dogs could also be used to characterize the respiratory compromise in those dogs [5]. Due to the complexity of these tests, polisomnography and plethysmography were not available for our study.

Several authors suggested that cTnI determination in dogs is useful for identifying myocardial damage [38,39]. A large percentage of brachycephalic dogs $(47.8 \%)$ had high levels of cTnI. It is interesting to note that all (100\%) English bulldogs and 53.8\% of French bulldog had high levels of cTnI. The hypothesis is that, as occurs in humans with OSAS, BAOS can induce surges in sympathetic activity, hypoxia and increased blood pressure that lead to myocardial damage.

All patients included in the study were submitted to a thorough physical examination, blood work, thoracic $x$ ray and an electrocardiogram, and no evidence of cardiac disease was found. However brachycephalic dogs may be predisposed to pulmonary hypertension, due to chronic hypoxia, and dogs with pulmonary hypertension can have increased concentrations of cTnI [40]. A limitation of the study due to its multicentric nature is that echocardiography was not performed in all dogs and therefore pulmonary hypertension and occult cardiac disease cannot be completely ruled out as a cause of increase $\mathrm{cTnI}$ in some dogs from this study. Even though, the evaluation of ECG (presence of arrhythmias and 
tachycardia) and vertebral heart score (VHS) was evaluated in relation to $\mathrm{CTnI}$, CRP and $\mathrm{Hp}$ without obtaining any association. Moreover cTnI levels can vary with age and breed $[38,41,42]$ and canine reference intervals must be established for different age groups and breeds. This information should be added to future studies. Although further studies must clarify this condition, the results obtained from this study suggest that some patients with BAOS had hidden but biochemically detectable myocardial injury. Even though, it must be considered that repeated measurements of those biomarkers levels in each individual dog could have increased the robustness of the results. On the other hand, the lack of a control group is an important limitation of the study since could have helped to interpret the results. But it must be taken to account, that is very difficult to find brachycephalic dogs with a normal breathing pattern because nearly all of them have some degree of upper airway obstruction.

\section{Conclusions}

To conclude, this study suggests that BAOS does not induce an evident systemic inflammatory status according to values of CRP and $\mathrm{Hp}$. On the other hand, these preliminary observations suggest that some dogs with BAOS may suffer some grade of myocardial damage that could be detected by cTnI levels. Further studies including more animals are required to define the predictive value of those biomarkers in dogs with BAOS. To the authors' knowledge, this is the first description of CRP, $\mathrm{Hp}$ and cTnI levels in dogs with BAOS.

\section{Methods}

This multicentre study is based on a prospective evaluation of brachycephalic dogs over a two-year period (January 2008-January 2010) in four veterinary hospitals (Hospital Clinic Veterinari, Barcelona; ARS Veterinaria, Barcelona; Centro Policlínico Veterinario El Raspeig, Alicante and Centre Hospitalier Vétérinaire Frégis, Arcueil). The study group consisted of brachycephalic dogs presented for the diagnosis of BAOS. In order to be included in the study dogs must be brachycephalic with clinical signs of upper airway obstruction, with a body condition from $4 / 9$ to $7 / 9$, without any previous treatment for BAOS and any present medication. On the other hand, dogs included in the study must not present any other illness apart from BAOS.

\section{Ethical authority}

The procedures conducted for this study were absolutely designed to improve the welfare of dogs. Moreover, owners were exhaustively informed of the blood work and exploratory procedures applied on each animal, and signed an authorization file of acceptance. On the other hand, the study was evaluated and accepted by the
Ethical Committee on Animal and Human Research (CEEAH) of the Universitat Autònoma de Barcelona (UAB).

\section{Clinical evaluation}

Upon admission, a physical examination, thoracic radiography and an electrocardiogram were performed on all patients. Moreover cell blood count, serum biochemistry and serum levels of CRP, Hp and cTnI were evaluated. A complete clinical form was obtained from each patient, designed to homogenize the information between centers. The frequency of upper respiratory signs (stertors, snoring, inspiratory effort, exercise intolerance and syncope) and digestive signs (ptyalism, regurgitation and vomiting) were recorded. On the basis of the frequency of each respiratory and digestive sign, a global classification of four grades was obtained: absent (grade 0), mild (grade 1), moderate (grade 2) and severe (grade 3 ).

Diagnosis of all anatomical components of BAOS was made via oropharyngeal and laryngeal examination and thoracic radiographs in anesthetized dogs. Premedication included $0.05 \mathrm{mg} / \mathrm{kg}$ acepromacine and $0.01 \mathrm{mg} / \mathrm{kg}$ buprenorfine intramuscularly. Anaesthesia was induced with propofol. A soft palate that extended beyond its contact with the epiglottis, or that extended caudally to distal poles of the tonsils, was considered redundant. BAOS diagnosis was carried out on the basis of both upper airway respiratory signs and anatomic abnormalities, as has been described elsewhere [25].

Lateral thoracic radiography aimed at measuring the vertebral heart score and ratio of the thoracic inlet (DTI) to the tracheal diameter (DT) was performed anesthetized animals in order to detect tracheal hypoplasia, defined as DTI/DT ratio < $0.16[22,34]$. The findings of physical examinations, cardiac auscultation, electrocardiographic and radiographic abnormalities, and blood tests provided no evidence of any other diseases in patients included in the study.

\section{Biomarker analysis}

Blood samples were collected in $5 \mathrm{~mL}$ serum blood tubes and centrifuged within 30 minutes after collection. The serum obtained was separated and stored at $-80{ }^{\circ} \mathrm{C}$ for biomarker analysis. Sample storage varied from 1 to 2 months. Samples were sent using dry ice and were thawed only once, at the time of analysis.

CRP concentration was measured using a human immunoturbidimetric assay ${ }^{\mathrm{a}}$ that showed a correlation of 0.98 with a specific canine ELISA assay ${ }^{\mathrm{b}}$ which has been validated for use in dogs [43]. A pooled canine serum sample with high concentration of CRP measured by the specific canine ELISA assay was used as standard. $\mathrm{Hp}$ concentrations were measured by a commercially available colorimetric method $^{\mathrm{c}}$, previously validated in 
dogs [43]. CRP and Hp levels were measured in serum using an automated biochemistry analyzer ${ }^{\mathrm{d}}$ and had intra-run and inter-run coefficients of variation $<10 \%$. cTnI was determined by the DPC Immulite, a previously validated assay that gives satisfactory imprecision values [19]. CRP, Hp and cTnI levels were classified as normal or high according to our laboratory reference values (normal values: $\mathrm{CRP}<20 \mathrm{mg} / \mathrm{L} ; \mathrm{Hp}<3 \mathrm{~g} / \mathrm{L} ; \mathrm{cTnI}<0.05 \mathrm{ng} / \mathrm{ml}$ ).

\section{Statistical analysis}

The statistical analysis was performed using the statistical software SPSS statistics ${ }^{\mathrm{e}}$. A value of $p<0.05$ was used to determine significance. Data were tested for normality by Shapiro-Wilk and Kolmogorov-Smirnov tests. Most data did not follow a normal distribution and nonparametric tests were employed. A Spearman Rho test (Table 2) was used to search for a correlation between the degree of clinical signs and all the studied parameters. Two variables relating to the severity of clinical signs were introduced into the database: grade of respiratory signs and grade of digestive signs. Grades 1, 2 and 3 for respiratory signs were considered for analysis. All grades were used for digestive signs. The KruskallWallis test was employed to compare values for a single variable between three or more groups and differences between groups were compared with rank sum (MannWhitney U) tests.

\section{Endnotes}

${ }^{\mathrm{a}}$ CRP OSR 6147 Olympus Life and Material Science Europe GmbH, Lismeehan, O'Callaghan's Mills, Co. Clare, Ireland

${ }^{\mathrm{b}}$ Tridelta Phase range canine CRP kit, Tridelta Development Ltd, Brey, Ireland

${ }^{\mathrm{c}}$ Tridelta Phase range haptoglobin kit, Tridelta Development Ltd.

${ }^{\mathrm{d} O l y m p u s} 2700$ Automatic Chemistry Analyzer, Olympus Europe GmbH, Hamburg, Germany

${ }^{\mathrm{e}} \mathrm{SPSS} 17.0$ version, Chicago, IL, USA

\section{Abbreviations}

BAOS: Brachycephalic upper airway obstructive syndrome; CRP: C-reactive protein; CTnl: Cardiac troponin I; DTI/DT: Thoracic inlet to tracheal diameter; ECG: Electrocardiogram; Hp: Haptoglobin; OSAS: Obstructive sleep apnea syndrome; VHS: Vertebral heart score.

\section{Competing interests}

The authors declare that they have no competing interests.

\section{Authors' contribution}

The experiments were conceived by MP, RC and JL. JP participated in the design of the study and performed the statistical analysis. MDT, CB, CP and JMC have made substantial contributions on the acquisition of data used in the manuscript and have participated on the interpretation of data. JJC have made all the biomarker analysis of the animals studied. All authors contributed to, and approved the final manuscript.

\section{Acknowledgements}

Mr. Artur Font and all the veterinarians who work at the Hospital Clinic Veterinari de la Universitat Autònoma de Barcelona are acknowledged for technical assistance during data collection.

This manuscript forms part of a thesis submitted by Dra. Marta Planellas to the Autonomous University of Barcelona (UAB), Animal Medicine and

Surgery Department.

\section{Author details}

${ }^{1}$ Animal Medicine and Surgery Department. Faculty of Veterinary Medicine, Universitat Autònoma de Barcelona (UAB), Cerdanyola del Vallès, Barcelona 08193, Spain. ${ }^{2}$ Centro Policlínico Veterinario Raspeig, Camino Rodalet no 17 , Sant Vicente del Raspeig, Alicante 03690, Spain. ${ }^{3}$ Centre Hospitalier Vétérinaire Frégis, Av. Aristide Briand no 43, Arcueil 94110, France. ${ }^{4}$ Hospital ARS Veterinària, Cardedeu str. no 3, Barcelona 08023, Spain.

${ }^{5}$ Otorrinolaringology service in the Hospital General Universitari de la Vall d'Hebron, Passeig de la Vall d'Hebron no 119-129, Barcelona 08035, Spain. ${ }^{6}$ Department of Animal Medicine and Surgery, Faculty of Veterinary Medicine, Regional Campus of International Excellence "Campus Mare Nostrum" Murcia University, Espinardo, Murcia 30100, Spain.

Received: 29 February 2012 Accepted: 7 August 2012

Published: 31 August 2012

\section{References}

1. Wykes PM: Brachycephalic airway obstructive syndrome. Probl Vet Med 1991, 3:188-197.

2. De Lorenzi $D$, Bertoncello D, Drigo M: Bronchial abnormalities found in a consecutive series of 40 brachycephalic dogs. J Am Vet Med Assoc 2009, 235:835-840.

3. Poncet CM, Dupré GP, Freiche VG, Bouvy BM: Long-term results of upper respiratory syndrome surgery and gastrointestinal tract medical treatment in 51 brachycephalic dogs. I Small Anim Pract 2006 47:137-142

4. Ginn JA, Kumar MS, Mckiernan BC, Powers BE: Nasopharyngeal turbinates in brachycephalic dogs and cats. J Am Anim Hosp Assoc 2008, 44:243-249.

5. Bernaerts F, Talavera J, Leemans J, Hamaide A, Claeys S, Kirschvink N, Clercx C: Description of original endoscopic findings and respiratory functional assessment using barometric whole-body plethysmography in dogs suffering from brachycephalic airway obstruction syndrome. Vet J 2010, 183:95-102.

6. Riecks TW, Birchard SJ, Stephens JA: Surgical correction of brachycephalic syndrome in dogs: 62 cases. J Am Vet Med Assoc 2007, 230:1324-1328.

7. Pink JJ, Doyle RS, Hughes JM, Tobin E, Bellenger CR: Laryngeal collapse in seven brachycephalic puppies. J Small Anim Pract 2006, 47:131-135.

8. Hendricks JC, Kovalski R, Kline LR: Phasic respiratory muscle patterns and sleep - disordered breathing during rapid eye movement sleep in the English bulldog. Am Rev Resp Dis 1991, 144:1112-1120.

9. Hendricks JC, Petrof BJ, Panckeri K, Pack Al: Upper airway dilating muscle hyperactivity during non-rapid eye movement sleep in English bulldogs. Am Rev Resp Dis 1993, 1:185-194.

10. William A, Scharf SM: Obstructive sleep apnea, cardiovascular disease, and inflammation- is NF-Kappa $\beta$ the key? Sleep Breath 2007, 11:69-76.

11. Mills PJ, Dimsdale JE: Sleep apnea: a model for studying cytokines, sleep and sleep diruption. Brain Behav Immun 2004, 18:298-303.

12. Hartmann G, Tschop M, Fischer R, Bidlingmaier C, Riepl R, Tschöp K, Hautmann $H$, Endres $S$, Toepfer M: High altitude increases circulating interleukin-6, interleukin-1 receptor antagonist and C-reactive protein Cytokine 2000, 12:246-252.

13. Vgontzas AN, Zoumakis E, Bixler EO, Lin HM, Follett H, Kales A, Chrousos GP: Adverse effects of modest sleep restriction on sleepiness, performance, and inflammatory cytokines. J Clin Endoc Metab 2004, 89:2119-2126.

14. McGuire M, MacDermott M, Bradford A: The effects of chronic episodic hypercapnic hypoxia on rat upper airway muscle contractile properties and fiber-type distribution. Chest 2002, 122:1400-1406.

15. Bradford A, McGuire M, O'Halloran KD: Does episodic hypoxia affect upper airway dilator muscle function? Implications for the pathophysiology of obstructive sleep apnoea. Respir Physiol Neurobiol 2005, 147:223-234.

16. Kim JW, Lee S, In K, Kim J, You S, Kang K, Sim J, Lee S, Yoon D, Lee J, Shin C: Increase in serum haptoglobin and apolipoprotein $\mathrm{M}$ in patients with obstructive sleep apnoea. J Sleep Res 2009, 18:313-320. 
17. Adams JE, Bodor GS, Davila-Roman VG, Delmez JA, Apple FS, Ladenson JH, Jaffe AS: Cardiac troponin I: A marker with high specificity for cardiac injury. Circulation 1993, 88:101-106.

18. Babuin $L$, Jaffe AS: Troponin: the biomarker of choice for the detection of cardiac injury. Can Med Assoc J 2005, 173:1191-1202.

19. Apple FS, Murakami MM, Ler R, Walker D, York M: Analytical characteristics of commercial cardiac troponin I and T immunoassays in serum from rats, dogs, and monkeys with induced acute myocardial injury. Clin Chem 2008, 54:1982-1989.

20. Fonfara S, Loureiro J, Swift S, James R, Cripps P, Dukes-McEwan J: Cardiac troponin I as a marker for severity and prognosis of cardiac disease in dogs. Vet J 2010, 184:334-339.

21. Ricchiuti V, Sharkey SW, Murakami MM, Voss MM, Apple FS: Cardiac troponin I and T alteration in dogs hearts with myocardial infarction: Correlation with infarct size. Am J Clin Pathol 1998, 110:241-247.

22. Buchanan JW: Vertebral scale system to measure heart size in radiographs. Vet Clin North Am Small Anim Pract 2000, 30:379-393.

23. Hendricks JC, Kline LR, Kovalski RJ, O'Brien JA, Morrison AR, Pack Al: The English bulldog: a natural model of sleep-disordered breathing. J Appl Physiol 1987, 63:1344-1350.

24. Petrof BJ, Pack Al, Kelly AM, Eby J, Hendricks JC: Pharyngeal myopathy of loaded upper airway in dogs with sleep apnea. J Appl Physiol 1994, 76:1746-1752.

25. Hendricks JC: Brachycephalic airway syndrome. Vet Clin North A Small Anim Pract 1992, 22:1145-1153.

26. Bright RM, White RAS: Brachycephalic airway obstruction syndrome. A review of 118 cases. Can Pract 1997, 22:18-21.

27. Poncet CM, Dupré GP, Freiche VG, Estrada MM, Poubanne YA, Bouvy VM Prevalence of gastrointestinal tract lesions in 73 brachycephalic dogs with upper respiratory syndrome. J Small Anim Pract 2005, 46:273-279.

28. Fasanella FJ, Shivley JM, Wardlaw JL, Givaruangsawat S: Brachycephalic airway obstructive syndrome in dogs: 90 cases (1991-2008). J Am Vet Med Assoc 2010, 237:1048-1051.

29. Torrez CV, Hunt GB: Results of surgical correction of abnormalities associated with brachycephalic airway obstruction syndrome in dogs in Australia. J Small Anim Pract 2006, 47:150-154.

30. Coyne BE, Fingland RB: Hypoplasia of the trachea in dogs: 103 cases (1974-1990). J Am Vet Med Assoc 1992, 201:768-772.

31. Bedford PGC: Tracheal hypoplasia in the English bulldog. Vet Rec 1982, 11:58-59.

32. Hendricks JC: Brachycephalic airway syndrome. In Textbook of respiratory disease in dogs and cats. Edited by King LG. Philadelphia: Saunders; 2004:310-318.

33. Mason RA: Tracheal hypoplasia. In Textbook of respiratory disease in dogs and cats. Edited by King LD. Philadelphia: Saunders; 2004:356-359.

34. Harvey CE, Fink EA: Tracheal diameter: analysis of radiographic measurements in brachycephalic and non-brachycepalic dogs. J Am Anim Hosp Assoc 1982, 18:570-576.

35. Schamsuzzaman ASM, Winnicki M, Lanfranchi P, Wolk R, Kara T, Accurso V, Somers VK: Elevated C-reactive protein in patients with obstructive sleep apnea. Circulation 2002, 105:2462-2464.

36. Yokoe T, Minoguichi K, Matsuo H, Oda N, Minoguchi H, Yoshino G: Elevated levels of C-reactive protein and interleukin- 6 in patients with obstructive sleep apnea syndrome are decreased by nasal continuous positive airway pressure. J Am Heart Assoc 2003, 107:1129-1134.

37. Rancan L, Romussi S, Albertini M, García P, Vara E, Sánchez de la Muela M: Circulating proinflammatory cytokines and nitric oxide are increased in brachycephalic dogs. In Proceedings 20th European Congress of Veterinary Surgery, 7-9th July; Belgium. Edited by Stephen B.: ; 2011:151

38. Oyama MA, Sisson DD: Cardiac troponin I concentration in dogs with cardiac disease. J Vet Intern Med 2004, 18:831-839.

39. Spratt DP, Mellanby RJ, Drury N, Archer J: Cardiac troponin I: evaluation of a biomarker for the diagnosis of heart disease in the dog. J Small Anim Pract 2005, 46:139-145

40. Guglielmini C, Civitella C, Diana A, Di Tommaso M, Cipone M, Luciani A: Serum cardiac troponin I concentration in dogs with precapillary and postcapillary pulmonary hypertension. J Vet Intern Med 2010, 24:145-152

41. O'Brien PJ, Smith DE, Knechtel TJ, Marchak MA, Pruimboom-Brees I, Bree DJ, Spratt DP, Archer FJ, Butler P, Potter AN, Provost JP, Richard J, Snyder PA, Reagan WJ: Cardiac Troponin I is a sensitive, specific biomarker of cardiac injury in laboratory animals. Lab Anim 2006, 40:153-171.
42. Baumwar RD, Orvalho J, Meurs KM: Evaluation of serum cardiac troponin I concentration in Boxers with arrhythmogenic right ventricular cardiomyopathy. Am J Vet Res 2007, 68:524-528.

43. Martínez S, Cerón JJ: Validation of commercial assays for the determination of haptoglobin, C-reactive protein and serum amyloid $\mathrm{A}$ in dogs. Arch Med Vet 2005, 37:61-66.

doi:10.1186/1746-6148-8-152

Cite this article as: Planellas et al.: Evaluation of C-reactive protein,

Haptoglobin and cardiac troponin 1 levels in brachycephalic dogs with upper airway obstructive syndrome. BMC Veterinary Research 2012 8:152.

\section{Submit your next manuscript to BioMed Central and take full advantage of:}

- Convenient online submission

- Thorough peer review

- No space constraints or color figure charges

- Immediate publication on acceptance

- Inclusion in PubMed, CAS, Scopus and Google Scholar

- Research which is freely available for redistribution

Submit your manuscript at www.biomedcentral.com/submit
C Biomed Central 